\title{
Predictors of severity and mortality of COVID-19 at a tertiary care center in a Lower-middle income country
}

\author{
Nosheen Nasir ${ }^{1}$, Kiren Habib ${ }^{1}$, Iffat Khanum ${ }^{1}$, Naveera Khan ${ }^{1}$, Zehra Abdul Muhammad ${ }^{1}$, \\ and Syed Faisal Mahmood ${ }^{1}$ \\ ${ }^{1}$ Aga Khan University
}

October 1, 2020

\begin{abstract}
Objective COVID-19 mortality and outcomes differ significantly across the globe. Limited data exists from low-middle income countries (LMICs) on risk-factors for COVID-19 severity and mortality. We describe the clinical spectrum and predictors of mortality and severity of illness in COVID-19 from a single center in Karachi, Pakistan. Methods Retrospective cohort study of adults admitted with COVID-19 between February-June 2020 were reviewed and logistic regression applied on admission related risk-factors for severity and mortality. Results A total of 445 patients [66.97\% males, mean age 51.6 (18-91) years] were admitted with PCR confirmed COVID-19 during the study period. Asymptomatic and severe/critical disease occurred in $55(12.36 \%)$ and $137(30.79 \%)$ patients, respectively. The proportion of severe disease increased with time and most (268, $60.22 \%$ ) had [?] 1 co-morbid. Disease severity was associated with age [?] 60 (OR:1.92), shortness of breath (OR:4.43) , CRP [?]150mg/L (OR:1.77), LDH [?] 500 I.U/L (OR:1.98), Neutrophil to Lymphocyte ratio (NLR) [?]5 (OR:2.80) and unit increase in serum creatinine (OR:1.32). All-cause mortality was $13 \%$. Mortality was associated with septic shock $(\mathrm{AOR}=13.2)$, age [?] 60 (AOR: 3.25), Ferritin [?] 1500ng/ml (AOR: 3.78) and NLR [?] 5 (AOR: 4.04). Conclusion We describe the experience with COVID-19 from a tertiary-care hospital in a LMIC. Our study found a comparatively low inpatient mortality, high proportion of diabetics, and neutrophil to lymphocyte ratio of greater than 5 as a predictor of both severity of illness and as poor prognostic marker in COVID-19.
\end{abstract}

Category of paper: Original Article

Title: Predictors of severity and mortality of COVID-19 at a tertiary care center in a Lower-middle income country

Abstract

Objective

COVID-19 mortality and outcomes differ significantly across the globe. Limited data exists from low-middle income countries (LMICs) on risk-factors for COVID-19 severity and mortality. We describe the clinical spectrum and predictors of mortality and severity of illness in COVID-19 from a single center in Karachi, Pakistan.

Methods

Retrospective cohort study of adults admitted with COVID-19 between February-June 2020 were reviewed and logistic regression applied on admission related risk-factors for severity and mortality.

Results

A total of 445 patients [66.97\% males, mean age 51.6 (18-91) years] were admitted with PCR confirmed COVID-19 during the study period. Asymptomatic and severe/critical disease occurred in 55 (12.36\%) and 
$137(30.79 \%)$ patients, respectively. The proportion of severe disease increased with time and most (268, $60.22 \%)$ had [?] 1 co-morbid. Disease severity was associated with age [?] 60 (OR:1.92), shortness of breath (OR:4.43), CRP [?]150mg/L (OR:1.77), LDH [?] 500 I.U/L (OR:1.98), Neutrophil to Lymphocyte ratio (NLR) [?]5 (OR:2.80) and unit increase in serum creatinine (OR:1.32). All-cause mortality was $13 \%$. Mortality was associated with septic shock $(\mathrm{AOR}=13.2)$, age [?] 60 (AOR: 3.25), Ferritin [?] 1500ng/ml (AOR: 3.78) and NLR [?] 5 (AOR: 4.04).

\section{Conclusion}

We describe the experience with COVID-19 from a tertiary-care hospital in a LMIC. Our study found a comparatively low inpatient mortality, high proportion of diabetics, and neutrophil to lymphocyte ratio of greater than 5 as a predictor of both severity of illness and as poor prognostic marker in COVID-19.

Keywords:

COVID-19; Mortality; Pakistan;

What is known about this topic?

1. COVID-19 mortality and outcomes differ significantly across the globe.

2. Pakistan's COVID-19 fatality rate of $2.13 \%$ is significantly lower than that reported from developing countries.

3. Factors predictive of severity of illness and mortality have varied and there is limited data from low and middle income countries although these countries differ in disease burden as well as in prevalence of co-morbids such as Diabetes and have poor infrastructure to deal with this pandemic.

What does this article add?

1. There is under-representation of data on outcomes of COVID-19 from these LMICs in international literature despite substantial disease burden in these countries.

2. This is the first retrospective cohort study from this country which was conducted to determine predictors of severity of illness and poor prognostic markers in COVID-19 on 445 patients.

3. Outcome assessment for mortality as well as other complications including development of Acute Respiratory Distress Syndrome, Nosocomial infections and acute kidney injury was performed and poor prognostic markers were determined.

4. We found a comparatively lower mortality despite greater prevalence of diabetes in our cohort and we found that a neutrophil to lymphocyte ratio of greater than 5 at presentation can be used as a marker to predict greater severity of illness and mortality

Introduction

The World Health Organization (WHO) declared a Coronavirus Disease 2019 (COVID-19) a global pandemic on March 11, 2020, and since then it has affected 213 countries and territories worldwide. Pakistan currently ranks at number 16 with greater than 250,000 reported cases and 6000 mortalities (1). COVID-19 displays a spectrum of presentations ranging from asymptomatic to fatal. While the causative organism, Severe Acute Respiratory Syndrome Coronavirus-2, has a tropism for multiple organ systems (2), pulmonary manifestations predominate. Cough, fever, shortness of breath, myalgia, and headaches are some of the most commonly reported symptoms (3). For management and research, the World Health Organization categorizes disease severity into mild (symptomatic without evidence of pneumonia or hypoxia), moderate (with clinical signs of pneumonia and oxygen saturation $>=90 \%$ on room air), severe (signs of pneumonia with respiratory rate $>30$ breaths/min or severe respiratory distress or SpO2 $<90 \%$ on room air) and critical (development of Acute Respiratory Distress Syndrome or sepsis) (4) 
COVID-19 mortality and outcomes differ significantly across the globe. Six months into its COVID-19 outbreak, Pakistan, a low middle-income country, has crossed the peak of its epidemic. Pakistan's COVID19 fatality rate of $2.13 \%(5)$ is significantly lower than that reported in America and Europe, and lower than that of its neighbors Iran, Afghanistan, and China, but comparable to that of India (6). Sindh was the first Pakistani province to report a case of COVID-19. Since then it has had the highest number of cases $(43 \%)$ in the country (5), most from its largest city- the metropolitan Karachi. We report data on comorbidities, and clinical, biochemical, and radiological features and outcomes of COVID-19 in a larger cohort of 445 patients from our center-an academic, private, tertiary care hospital in Karachi with a dedicated facility for COVID-19.

Methods

\section{Study population and Design}

This retrospective cohort study included adult inpatients ([?]18 years old) from Aga Khan University Hospital, a 700 bedded tertiary academic medical center in Karachi Pakistan. All adult patients who were diagnosed with COVID-19 based on PCR positivity for SARS-Cov-2, and those who died or were discharged between Feb, 2020 and June, 2020, were included in our study.

Ethics approval

This study received approval from the Aga Khan University Ethics Review Committee of the hospital (ERC reference number: 2020-3650-11773). The data was collected from hospital records and the requirement for informed consent was waived by the hospital ethical review committee as data was anonymized and no personal identifiers were collected.

\section{Data collection}

Demographics, clinical characteristics, outcomes and treatment details of confirmed COVID-19 patients including underlying co-morbidities, laboratory and radiological investigations, and complications during hospitalization were collected on a structured proforma from hospital medical records.

Diagnosis of COVID-19

Nasopharyngeal swabs were processed for detection of SARS-Cov-2 virus by real-time reverse transcriptase polymerase chain reaction (RT-PCR) using the WHO protocol for the 2019- nCoV RT-PCR assay in March 2020. Specimens in May were tested using the Cobasß SARS-CoV-2 RT-PCR assay (Roche Diagnostics, USA). Radiological diagnosis of pneumonia was made by evaluation of infiltrates observed on chest radiographs and/or CT chest. A multidisciplinary team of doctors including infectious disease consultants, pulmonologists, and intensivists was involved in the identification of cases and their management.

\section{Statistical analysis}

Descriptive analysis was performed for demographic features with mean and standard deviation or median with interquartile range (IQR) reported for quantitative variables such as age and lengths of hospital stay as appropriate. Frequencies (percentage) for qualitative variables such as sex, co-morbid conditions, mortality, and complications. Continuous variables were also transformed into categorical variables for further analysis as indicated. $\chi 2$ test of independence or Fischer exact test was performed for categorical variables such as comparison of those with COVID-19 who died with those who were discharged. Univariable and multivariable ordinal logistic regression was performed to determine the risk factors associated with illness severity of COVID-19, (mild, moderate, and severe/critical). Multivariable logistic regression analysis was performed on variables found to be significant on univariate analysis to identify factors associated with death in COVID19 infection. All p value [?]0.05 was taken as significant. STATA ver 12.1was used for data analysis.

Results

Demographic, Clinical characteristics and course of the disease 
A total of 11393 adult patients were admitted at the Aga Khan University Hospital between Feb $26^{\text {th, }} 2020$, and June $10^{\text {th, }} 2020$. Out of this, 445 patients were diagnosed with COVID-19 based on RT-PCR positivity for SARS-CoV-2. Most of those admitted were males $(298,66.97 \%)$ and had a mean age of 51.6 (18-91) years. Asymptomatic disease occurred in $55(12.36 \%)$ of the patients, while severe/critical disease occurred in $137(30.79 \%)$. Most $(268,60.22 \%)$ patients had at least one or more than one co-morbidity, of which hypertension was the most common $(37.5 \%)$ followed by diabetes (36.4\%) (Table 1). Admissions peaked in May and then steadily reduced over time. The proportion of patients with severe disease increased with time with $25 \%$ of patients admitted with severe disease in March and $34 \%$ of patients admitted with severe disease in May (Figure 1).

In those who were symptomatic, the most common symptom was fever $(80 \%)$ followed by cough $(61.3 \%)$ and shortness of breath (61.0\%). The median duration of illness prior to presentation was 7 days (IQR $3-10$ ) and was longer with worsening severity of illness (median of 3 days in mild, 7 days in moderate, and 7 days in severe respectively). Chest X-rays were performed in almost all patients (97\%). Chest X-rays were normal in $21.57 \%$ of patients. Unilateral involvement was seen less frequently as compared to bilateral disease ( 7.69 versus $66 \%$ respectively). Median C - reactive protein level was $83.3 \mathrm{mg} / \mathrm{L}$ (IQR: $27.8-178.9$ ) and the median Ferritin level was $551 \mathrm{ng} / \mathrm{ml}$ (IQR: 254.1-1258.3). Overall, 124 (27.9\%) required non-invasive ventilation (NIV) and $64(14.5 \%)$ required invasive positive pressure (IPPV) ventilation. Treatment received varied as the protocols changed with chloroquine use dropping from $2.25 \%$ of patients to zero between February and April. Similarly, hydroxychloroquine was given to $31 \%$ of patients overall with use declining from $47 \%$ to $2.1 \%$ between April and June. Septic shock was seen in $62(14 \%)$ of the cases while $44(9.9 \%)$ presented in multi-organ dysfunction. Acute kidney injury was seen in $96(21.62 \%)$ and $46(10.34 \%)$ patients presented with a myocardial infarction alone. Secondary infections were common and occurred in 59 (13.3\%) of patients. A total of 58 died with an overall mortality rate of $13 \%$. (Table 1).

\section{Determinants of Severity of illness}

Patients were classified as having asymptomatic/mild disease in $35 \%$, moderate in $34 \%$, or severe/critical disease in $30 \%$ based on WHO criteria. Mean age was significantly higher in patients with severe disease (59 years) compared to mild (42 years) and there was a male preponderance throughout all the levels of severity of illness. The presence of comorbidities such as diabetes, hypertension, and ischemic heart disease was greater in those with severe disease compared to patients with mild and moderate disease (p-value $<0.001)$. A greater proportion of patients with severe disease had shortness of breath (83\%) on presentation. Chest radiographic findings were bilateral patchy infiltrates in the majority of patients with moderate $(87.5 \%)$ and severe disease $(92 \%)$ whereas the majority of mildly diseased patients $(60 \%)$ had a normal chest x-ray. Patients with greater severity of illness had significantly higher median CRP, Ferritin, LDH, and D-Dimer values as well as mean neutrophil to lymphocyte ratio compared to those with mild and moderate disease (pvalue $<0.001)$. Most patients received supplemental oxygen in moderate and severe disease $(88 \%$ and $98.5 \%$ respectively). Steroids were used in the majority of patients with moderate and severe disease (79\% and $90 \%$ respectively). Tocilizumab was given to $86(19.3 \%)$ of cases and predominantly in those with severe disease, where almost half $(47.4 \%)$ received this. Antibiotic use was common in severe disease with $91 \%$ receiving any antibiotics as opposed to 16.5 of the mild and $57 \%$ of moderate cases. Ordinal logistic regression was performed to determine predictors of severity (Table 2). Multivariable ordinal regression analysis revealed that the risk of having severe disease was 1.92 (95\% CI: 1.23 -3.03) times higher in patients with age greater than or equal to 60 years of age when compared with patients of less than 60 years. Presence of shortness of breath at presentation ( $\mathrm{OR}=4.43 ; 95 \% \mathrm{CI}: 2.73-7.22)$ and presence of bilateral patchy infiltrates on chest radiograph $(\mathrm{OR}=5.81 ; 95 \% \mathrm{CI}$ : 2.90-11.62) was significantly associated with greater severity of illness. Among laboratory investigations done at admission; the risk of greater severity of illness was associated with $\mathrm{CRP}$ of greater than or equal to $150 \mathrm{mg} / \mathrm{L}(\mathrm{OR}=1.77 ; 95 \% \mathrm{CI}: 1.10-2.85), \mathrm{LDH}$ of greater than or equal to 500 I.U/L (OR=1.98; 95\% CI: 1.25-3.16), Neutrophil to Lymphocyte ratio greater than or equal to $5(\mathrm{OR}=2.80$; 95\% CI: 1.77-4.42)and unit increase in serum creatinine level in $\mathrm{mg} / \mathrm{dl}$ (OR=1.32; 95\% CI: 1.07-1.61).

Factors associated with risk of death 
Logistic regression analysis was performed to determine factors associated with mortality in patients with COVID-19. In the univariate analysis, mortality was significantly associated with older age, male sex, comorbid conditions, and presence of complications such as septic shock, multi-organ dysfunction, acute kidney injury, myocardial infarction, and nosocomial infection (Table 3). However, presence of septic shock (AOR= 13.2; 95\% CI: 3.78-46.65), Multi-organ dysfunction (AOR=8.6 (95\%CI: 2.08- 35.64), presence of acute kidney injury $(\mathrm{AOR}=5.52 ; 95 \% \mathrm{CI}: 1.78-17.06)$, admission to the intensive care unit $\mathrm{ICU})(\mathrm{AOR}=3.99 ; 95 \% \mathrm{CI}$ : $1.22-13)$, age of greater than or equal to 60 years $(\mathrm{AOR}=3.25 ; 95 \% \mathrm{CI}: 1.07-9.89)$ and among laboratory investigations; serum Ferritin of greater than or equal to $1500 \mathrm{ng} / \mathrm{ml}$ (AOR=3.78; 95\%CI: $1.21-11.8$ ) and NLR of greater than or equal to $5(\mathrm{AOR}=4.04 ; 95 \% \mathrm{CI}: 1.14-14.35)$ were independently associated with mortality in the multivariable logistic regression analysis after adjusting for confounding and interactions.

Discussion

Our study reports several notable findings. First, while our cohort had a comorbid prevalence comparable to, or higher than that reported by other centers, no comorbid was found to have a statistically significant association with disease severity on multivariate analysis. This is in contrast to global reports of the association between several comorbid and COVID-19, including Ischemic Heart Disease, Diabetes Mellitus, Hypertension, cancer, Chronic Lung Disease, and Cerebrovascular Disease (7,8). Limited studies in the literature report a similar absence of statistically significant association (9) (10). It is possible that baseline control of comorbid conditions may influence COVID-19 outcomes. For instance, uncontrolled inpatient hyperglycemia, with or without known diabetes, is an independent predictor of worse outcomes (11) (12) (13). Data on baseline and inpatient comorbid control was not collected in our study. Future analyses of the association of outcomes with comorbid conditions should be stratified according to comorbid control to better describe the possible relationship.

Second, on multivariate analysis of laboratory parameters at presentation, only the NLR was found to have a statistically significant association with both disease severity and mortality. C-Reactive protein, Lactate Dehydrogenase, and Creatinine were found to be associated with disease severity alone, whereas ferritin was associated with mortality alone. A trend of higher levels of biochemical and hematological markers of inflammation and organ dysfunction with increasing disease severity and mortality was also observed, consistent with literature from other centers (14). However, our study supports the early use of NLR as a single marker for risk stratification for both disease progression and mortality, making this cost-effective and readily available tool especially valuable in resource-limited settings.

The case fatality rate from Pakistan has remained around 2-3\% which is considerably lower than Italy and Iran but similar to CFR reported from China and India (1). In this study, in-hospital mortality was $13.88 \%$, and the mean length of hospitalization was 7.37 days. In-hospital mortality has been reported to be $28 \%$ from tertiary care centers in China (15), 21.7\% from centers in New York (16), 20\% from Iran (17), and $53.4 \%$ in-hospital mortality from ICUs in Lombardy, Italy (18). To better characterize differences in mortality, we determined the risk factors for death among hospitalized patients. Our study showed that multi-organ dysfunction, septic shock, and admission to intensive care unit on presentation were associated with mortality. These are similar to risk factors reported from various regions across the globe, though individual risk factors have varied (16) (19). Acute kidney injury was also one of the independent predictors of mortality and has been reported from various countries as well (19). Most studies found an independent association with comorbid conditions such as diabetes, chronic kidney disease, and malignancy $(16)(20,21)$. However, although these were significant on univariable analysis, these were not found to be independent predictors of death on multivariable analysis. This is quite interesting considering the greater proportion of diabetics in our cohort (36\%) compared to (7.5 to 19\%) from hospitalized COVID-19 patients in China and $13 \%$ from Italy. This is similar if we consider lower CFR from South Asia despite the greater prevalence of Diabetes in this region and possible reasons could be multifactorial including epigenetic and lifestyle differences (22) (23).

Fourth, compared to studies that have shown increased age and male sex to be risk factors for an adverse outcome (24), our study found an association of mortality with advanced age of greater than or equal to 60 
years, but we did not find an association of mortality with male sex on multivariable analysis. To understand this further, we looked at mortality sex ratios across all age groups and found that while it was 7.6 for the age interval 50-70 years, it was 2.3 for the age interval of 70-90 years. This highlights the need for disaggregated data to better understand the interaction between biological sex and age and its association with mortality (25). Among laboratory investigations we found the association of increased NLR and high serum ferritin level to be independent predictors of mortality which is consistent with literature reported from other studies and hence can be used to identify patients at risk early in course of disease $(9)(26,27)$.

Also noteworthy is the lack of mortality benefit from any of the treatment modalities used. This includes the use of steroids in a large proportion of patients with moderate to severe disease, although a recently published clinical trial has shown benefit (28) in this population. Moreover, we found a greater incidence of nosocomial infections compared to other studies (29-31). Nosocomial infections were also associated with mortality in univariable analysis, which may be related to immunosuppression with tocilizumab and steroids. This may be an important observation in the context of low-middle income countries where the incidence of nosocomial infections is higher and can contribute to poor outcomes in COVID-19 (32).

Our study has several limitations including retrospective data collection and single-center experience that may limit generalizability. However, our study highlights important differences in factors associated with severity and mortality particularly relevant to a low-middle income country whereby despite a high prevalence of diabetes and increased incidence of nosocomial infections there was comparatively lower in-hospital mortality. It also emphasizes the key epidemiological differences in the nature of the outbreak experienced in our country for which we recommend validation by population-based studies.

\section{ABBREVIATIONS}

COVID-19: Coronavirus disease 2019

ARDS: Acute respiratory distress syndrome

CRP: C-reactive protein

ALT: Alanine aminotransferase

WHO: World health organization

CRS: Cytokine release syndrome

IL-6: Interleukin 6

IPPV: Intermittent positive pressure ventilation

NIV: Non-invasive ventilation

MDR: Multi-drug resistant

LMIC: Low-middle income country

Declarations

\section{Funding}

No funding was received for this study

\section{Conflicts of interest/Competing interests}

None of the authors have any conflict of interest

Ethics approval

This study was approved by the Ethics review committee of Aga Khan University Hospital (ERC reference number: 2020-3650-11773). 


\section{Consent to participate}

Due to the retrospective nature of the study, the Ethics Committee determined that no patient consent was required.

\section{Availability of data and material}

All data generated or analyzed during this study are included in this manuscript.

References

1. Worldometer. COVID-19 CORONAVIRUS PANDEMIC Reported Cases and Deaths by Country, Territory, or Conveyance 2020 [Available from: https://www.worldometers.info/coronavirus/.

2. Puelles VG, Lütgehetmann M, Lindenmeyer MT, Sperhake JP, Wong MN, Allweiss L, et al. Multiorgan and Renal Tropism of SARS-CoV-2. New England Journal of Medicine. 2020;383(6):590-2.

3. Stokes EK, Zambrano LD, Anderson KN, Marder EP, Raz KM, El Burai Felix S, et al. Coronavirus Disease 2019 Case Surveillance - United States, January 22-May 30, 2020. MMWR Morbidity and mortality weekly report. 2020;69(24):759-65.

4. World Health O. Clinical management of COVID-19: interim guidance, 27 May 2020 Geneva PP - Geneva: World Health Organization; 2020 [Available from: https://apps.who.int/iris/handle/10665/332196.

5. Government of P. COVID-19 Dashboard 2020 [Available from: http://covid.gov.pk/stats/pakistan.

6. World Health O. WHO Coronavirus Disease (COVID-19) Dashboard 2020 [Available from: https://covid19.who.int/?gclid=Cj0KCQjw6575BRCQARIsAMp-ksOrQWURza5_e_1n3oGnBZQuv4a8bJYDcobBzo1q7lqGidawLcK86JMaAthFEALw_wcB.

7. Zhou Y, Yang Q, Chi J, Dong B, Lv W, Shen L, et al. Comorbidities and the risk of severe or fatal outcomes associated with coronavirus disease 2019: A systematic review and meta-analysis. International Journal of Infectious Diseases. 2020;99:47-56.

8. Moccia F, Gerbino A, Lionetti V, Miragoli M, Munaron LM, Pagliaro P, et al. COVID-19-associated cardiovascular morbidity in older adults: a position paper from the Italian Society of Cardiovascular Researches. GeroScience. 2020;42(4):1021-49.

9. Huang S, Wang J, Liu F, Liu J, Cao G, Yang C, et al. COVID-19 patients with hypertension have more severe disease: a multicenter retrospective observational study. Hypertension Research. 2020;43(8):824-31.

10. Suleyman G, Fadel RA, Malette KM, Hammond C, Abdulla H, Entz A, et al. Clinical Characteristics and Morbidity Associated With Coronavirus Disease 2019 in a Series of Patients in Metropolitan Detroit. JAMA Network Open. 2020;3(6):e2012270-e.

11. Bode B, Garrett V, Messler J, McFarland R, Crowe J, Booth R, et al. Glycemic Characteristics and Clinical Outcomes of COVID-19 Patients Hospitalized in the United States. Journal of diabetes science and technology. 2020;14(4):813-21.

12. Wu J, Huang J, Zhu G, Wang Q, Lv Q, Huang Y, et al. Elevation of blood glucose level predicts worse outcomes in hospitalized patients with COVID-19: a retrospective cohort study. BMJ open diabetes research \& care. 2020;8(1).

13. Zhu L, She Z-G, Cheng X, Qin J-J, Zhang X-J, Cai J, et al. Association of Blood Glucose Control and Outcomes in Patients with COVID-19 and Pre-existing Type 2 Diabetes. Cell metabolism. 2020;31(6):106877.e3.

14. Khinda J, Janjua NZ, Cheng S, van den Heuvel ER, Bhatti P, Darvishian M. Association between markers of immune response at hospital admission and COVID-19 disease severity and mortality: A meta-analysis and meta-regression. Journal of medical virology. 2020:10.1002/jmv.26411-10.1002/jmv. 
15. Zhou F, Yu T, Du R, Fan G, Liu Y, Liu Z, et al. Clinical course and risk factors for mortality of adult inpatients with COVID-19 in Wuhan, China: a retrospective cohort study. Lancet (London, England). 2020.

16. Mikami T, Miyashita H, Yamada T, Harrington M, Steinberg D, Dunn A, et al. Risk Factors for Mortality in Patients with COVID-19 in New York City. Journal of General Internal Medicine. 2020.

17. Jalili M, Payandemehr P, Saghaei A, Sari HN, Safikhani H, Kolivand P. Characteristics and Mortality of Hospitalized Patients With COVID-19 in Iran: A National Retrospective Cohort Study. Annals of Internal Medicine. 2020.

18. Grasselli G, Greco M, Zanella A, Albano G, Antonelli M, Bellani G, et al. Risk Factors Associated With Mortality Among Patients With COVID-19 in Intensive Care Units in Lombardy, Italy. JAMA Internal Medicine. 2020.

19. Chen F, Sun W, Sun S, Li Z, Wang Z, Yu L. Clinical characteristics and risk factors for mortality among inpatients with COVID-19 in Wuhan, China. Clinical and Translational Medicine. 2020;10(2):e40-e.

20. Albitar O, Ballouze R, Ooi JP, Sheikh Ghadzi SM. Risk factors for mortality among COVID-19 patients. Diabetes Research and Clinical Practice. 2020;166.

21. Parohan M, Yaghoubi S, Seraji A, Javanbakht MH, Sarraf P, Djalali M. Risk factors for mortality in patients with Coronavirus disease 2019 (COVID-19) infection: a systematic review and meta-analysis of observational studies. The Aging Male. 2020:1-9.

22. Akhtar S, Nasir JA, Abbas T, Sarwar A. Diabetes in Pakistan: A systematic review and meta-analysis. Pak J Med Sci. 2019;35(4):1173-8.

23. Nanditha A, Ma RCW, Ramachandran A, Snehalatha C, Chan JCN, Chia KS, et al. Diabetes in Asia and the Pacific: Implications for the Global Epidemic. Diabetes Care. 2016;39(3):472 LP-85.

24. Williamson EJ, Walker AJ, Bhaskaran K, Bacon S, Bates C, Morton CE, et al. Factors associated with COVID-19-related death using OpenSAFELY. Nature. 2020;584(7821):430-6.

25. Bhopal SS, Bhopal R. Sex differential in COVID-19 mortality varies markedly by age. Lancet. 2020;396(10250):532-3.

26. Vargas-Vargas M, Cortes-Rojo C. Ferritin levels and COVID-19. Rev Panam Salud Publica. 2020;44:e72.

27. Liu Y, Du X, Chen J, Jin Y, Peng L, Wang HHX, et al. Neutrophil-to-lymphocyte ratio as an independent risk factor for mortality in hospitalized patients with COVID-19. J Infect. 2020;81(1):e6-e12.

28. Group RC, Horby P, Lim WS, Emberson JR, Mafham M, Bell JL, et al. Dexamethasone in Hospitalized Patients with Covid-19 - Preliminary Report. N Engl J Med. 2020.

29. He Y, Li W, Wang Z, Chen H, Tian L, Liu D. Nosocomial infection among patients with COVID-19: A retrospective data analysis of 918 cases from a single center in Wuhan, China. Infection control and hospital epidemiology. 2020;41(8):982-3.

30. Garcia-Vidal C, Sanjuan G, Moreno-García E, Puerta-Alcalde P, Garcia-Pouton N, Chumbita M, et al. Incidence of co-infections and superinfections in hospitalised patients with COVID-19: a retrospective cohort study. Clinical Microbiology and Infection. 2020.

31. Nori P, Cowman K, Chen V, Bartash R, Szymczak W, Madaline T, et al. Bacterial and fungal coinfections in COVID-19 patients hospitalized during the New York City pandemic surge. Infection control and hospital epidemiology. 2020:1-5.

32. Allegranzi B, Bagheri Nejad S, Combescure C, Graafmans W, Attar H, Donaldson L, et al. Burden of endemic health-care-associated infection in developing countries: systematic review and meta-analysis. Lancet (London, England). 2011;377(9761):228-41. 
Table 1: Comparison of mild, moderate and severe COVID-19 patients admitted at tertiary care center in Karachi, Pakistan

\begin{tabular}{|c|c|c|c|c|}
\hline Variables & All $(\mathrm{N}=445)$ & Mild $(n=156)$ & Moderate $(n=152)$ & Severe $(n=137)$ \\
\hline Age $($ mean $\pm \mathrm{SD})$ & $51.6 \pm 16.07$ & $42.34 \pm 16.23$ & $54.01 \pm 12.65$ & $59.36 \pm 14.14$ \\
\hline \multicolumn{5}{|l|}{ Sex } \\
\hline Male & $298(67 \%)$ & $86(55.1 \%)$ & $108(71.1 \%)$ & $105(76.6 \%)$ \\
\hline Female & $147(33 \%)$ & $70(44.9 \%)$ & $44(28.9 \%)$ & $32(23.4 \%)$ \\
\hline Duration of illness [median (IQR) days] & $7(3-10)$ & $3(0-7)$ & $7(5-10)$ & $7(5-10)$ \\
\hline \multicolumn{5}{|l|}{ Co-morbids } \\
\hline Diabetes & $162(36.4 \%)$ & $28(17.9 \%)$ & $70(46.1 \%)$ & $64(46.7 \%)$ \\
\hline Hypertension & $167(37.5 \%)$ & $30(19.2 \%)$ & $65(42.8 \%)$ & $72(52.6 \%)$ \\
\hline Ischemic heart disease & $74(16.6 \%)$ & $10(6.4 \%)$ & $25(16.4 \%)$ & $39(28.5 \%)$ \\
\hline Chronic kidney disease & $31(7 \%)$ & $2(1.3 \%)$ & $13(8.6 \%)$ & $16(11.7 \%)$ \\
\hline Malignancy & $22(4.9 \%)$ & $8(5.1 \%)$ & $5(3.3 \%)$ & $9(6.6 \%)$ \\
\hline Stroke & $10(2.2 \%)$ & $0(0 \%)$ & $3(2 \%)$ & $7(5.1 \%)$ \\
\hline Chronic liver disease & $8(1.8 \%)$ & $4(2.6 \%)$ & $1(0.7 \%)$ & $3(2.2 \%)$ \\
\hline \multicolumn{5}{|l|}{ Symptoms } \\
\hline Fever & $312(70.1 \%)$ & $83(53.2 \%)$ & $126(82.9 \%)$ & $103(75.2 \%)$ \\
\hline Cough & $239(53.7 \%)$ & $56(35.9 \%)$ & $103(67.8 \%)$ & $80(58.4 \%)$ \\
\hline Shortness of breath & $238(53.5 \%)$ & $24(15.4 \%)$ & $100(65.8 \%)$ & $114(83.2 \%)$ \\
\hline Sore throat & $48(10.8 \%)$ & $22(14.1 \%)$ & $18(11.8 \%)$ & $8(5.8 \%)$ \\
\hline Myalgias & $52(11.7 \%)$ & $21(13.5 \%)$ & $16(10.5 \%)$ & $15(10.9 \%)$ \\
\hline \multicolumn{5}{|l|}{ Radiologic findings } \\
\hline Normal & $96(21.6 \%)$ & $93(59.6 \%)$ & $3(2 \%)$ & $1(0.7 \%)$ \\
\hline Bilateral involvement & $291(65.4 \%)$ & $32(20.5 \%)$ & $133(87.5 \%)$ & $126(92 \%)$ \\
\hline Unilateral involvement & $34(7.6 \%)$ & $10(6.4 \%)$ & $17(11.2 \%)$ & $7(5.1 \%)$ \\
\hline Patchy infiltrates & $293(65.8 \%)$ & $33(21.2 \%)$ & $137(90.1 \%)$ & $123(89.8 \%)$ \\
\hline Multilobar involvement & $178(40 \%)$ & $14(9 \%)$ & $85(55.9 \%)$ & $79(57.7 \%)$ \\
\hline Consolidation & $48(10.8 \%)$ & $7(4.5 \%)$ & $18(11.8 \%)$ & $23(16.8 \%)$ \\
\hline CURB score [median (IQR)] & $1(0-2)$ & $0(0)$ & $1(0-1)$ & $2(1-3)$ \\
\hline \multicolumn{5}{|l|}{ Laboratory Investigations } \\
\hline C-Reactive Protein <150 mg/L & $252(56.6 \%)$ & $96(61.5 \%)$ & $105(69.1 \%)$ & $51(37.2 \%)$ \\
\hline C-Reactive Protein $>=150 \mathrm{mg} / \mathrm{L}$ & $193(43.4 \%)$ & $60(38.5 \%)$ & $47(30.9 \%)$ & $86(62.8 \%)$ \\
\hline $\mathrm{LDH}<500 \mathrm{IU} / \mathrm{L}$ & $235(52.8 \%)$ & $81(51.9 \%)$ & $109(71.7 \%)$ & $45(32.8 \%)$ \\
\hline $\mathrm{LDH}>=500 \mathrm{IU} / \mathrm{L}$ & $210(47.2 \%)$ & $75(48.1 \%)$ & $43(28.3 \%)$ & $92(67.2 \%)$ \\
\hline Neutrophil to lymphocyte ratio $<5$ & $255(57.3 \%)$ & $118(75.6 \%)$ & $95(62.5 \%)$ & $42(30.7 \%)$ \\
\hline Neutrophil to lymphocyte ratio $>=5$ & $190(42.7 \%)$ & $38(24.4 \%)$ & $57(37.5 \%)$ & $95(69.3 \%)$ \\
\hline Ferritin $<1500 \mathrm{ng} / \mathrm{ml}$ & $249(56 \%)$ & $84(53.8 \%)$ & $104(68.4 \%)$ & $61(44.5 \%)$ \\
\hline Ferritin $>=1500 \mathrm{ng} / \mathrm{ml}$ & $196(44 \%)$ & $72(46.2 \%)$ & $48(31.6 \%)$ & $76(55.5 \%)$ \\
\hline \multicolumn{5}{|l|}{ Type of Ward } \\
\hline ICU Admission & $68(15.3 \%)$ & $0(0 \%)$ & $1(0.7 \%)$ & $67(48.9 \%)$ \\
\hline SCU Admission & $148(33.3 \%)$ & $12(7.7 \%)$ & $75(49.3 \%)$ & $61(44.5 \%)$ \\
\hline Ward admission & $223(50.1 \%)$ & $141(90.4 \%)$ & $76(50 \%)$ & $6(4.4 \%)^{i}$ \\
\hline \multicolumn{5}{|l|}{ Treatment } \\
\hline Non-invasive ventilation & $124(27.9 \%)$ & $0(0 \%)$ & $35(23 \%)$ & $89(65 \%)$ \\
\hline Invasive ventilation & $64(14.4 \%)$ & $0(0 \%)$ & $0(0 \%)$ & $64(46.7 \%)$ \\
\hline Oxygen support & $278(62.5 \%)$ & $8(5.1 \%)^{i i}$ & $135(88.8 \%)$ & $135(98.5 \%)$ \\
\hline Antibiotics & $236(53 \%)$ & $25(16 \%)$ & $86(56.6 \%)$ & $125(91.2 \%)$ \\
\hline Azithromycin & $164(36.9 \%)$ & $16(10.3 \%)$ & $68(44.7 \%)$ & $80(58.4 \%)$ \\
\hline Hydroxychloroquine & $139(31.2 \%)$ & $11(7.1 \%)$ & $67(44.1 \%)$ & $61(44.5 \%)$ \\
\hline
\end{tabular}




\begin{tabular}{lllll}
\hline Variables & All $\mathbf{( N = 4 4 5 )}$ & Mild $\mathbf{( n = 1 5 6 )}$ & Moderate $\mathbf{( n = 1 5 2 )}$ & Severe $(\mathbf{n = 1 3 7})$ \\
\hline Chloroquine & $10(2.2 \%)$ & $1(0.6 \%)$ & $4(2.6 \%)$ & $5(3.6 \%)$ \\
Tocilizumab & $86(19.3 \%)$ & $0(0 \%)$ & $21(13.8 \%)$ & $64(46.7 \%)$ \\
Oseltamivir & $9(2 \%)$ & $0(0 \%)$ & $3(2 \%)$ & $6(4.4 \%)$ \\
Lopinavir/Ritonavir & $3(0.7 \%)$ & $0(0 \%)$ & $1(0.7 \%)$ & $2(1.5 \%)$ \\
Systemic steroids & $249(56 \%)$ & $6(3.8 \%)$ & $120(78.9 \%)$ & $123(89.8 \%)$ \\
Vasopressors & $59(13.3 \%)$ & $0(0 \%)$ & $1(0.7 \%)$ & $58(42.3 \%)$ \\
Complications & $122(27.4 \%)$ & $0(0 \%)$ & $19(12.5 \%)$ & $103(75.2 \%)$ \\
ARDS & $62(13.9 \%)$ & $0(0 \%)$ & $0(0 \%)$ & $62(45.3 \%)$ \\
Septic Shock & $44(9.9 \%)$ & $0(0 \%)$ & $3(2 \%)$ & $41(29.9 \%)$ \\
MODS & $59(13.3 \%)$ & $1(0.6 \%)$ & $10(6.6 \%)$ & $48(35 \%)$ \\
Nosocomial Infection & $96(21.6 \%)$ & $5(3.2 \%)$ & $21(13.8 \%)$ & $70(51.1 \%)$ \\
AKI & $46(10.3 \%)$ & $1(0.6 \%)$ & $9(5.9 \%)$ & $36(26.3 \%)$ \\
NSTEMI & $5(3-9)$ & $3(2-5)$ & $6(4-9)$ & $8(5-14)$ \\
Length of stay [median (IQR) days & $58(13 \%)$ & $0(0 \%)$ & $3(2 \%)$ & $55(40.1 \%)$ \\
Dead & $360(80.9 \%)$ & $144(92.3 \%)$ & $142(93.4 \%)$ & $74(54 \%)$ \\
Discharged & $27(6.1 \%)$ & $12(7.7 \%)$ & $7(4.6 \%)$ & $8(5.8 \%)$ \\
Left against medical advice (LAMA) & & &
\end{tabular}

1. These were patients with severe disease admitted for comfort care

2. This includes patient who had polytrauma, hematemesis, gastrointestinal bleed etc. who were incidentally diagnosed with COVID-19

3. These were varied, one with underlying malignancy, one with penumoperitoneum who was found on preop screening, a couple had raised ferritin only

Table 2: Multivariable ordinal logistic regression for factors associated with level of severity

\begin{tabular}{lllll}
\hline Variable & Categories & OR & 95\% CI & p-value \\
\hline Age & $<60$ years (Ref) & 1 & & \\
& $>=60$ years & 1.93 & $1.23-3.03$ & 0.004 \\
Shortness of breath & Absent (Ref) & 1 & & \\
\multirow{2}{*}{ Bilateral chest radiographic findings } & Present & 4.44 & $2.73-7.22$ & $<0.001$ \\
& Absent (Ref) & 1 & & \\
Patchy infiltrates & Present & 3.13 & $1.58-6.17$ & 0.001 \\
& Absent (Ref) & 1 & & \\
C-Reactive Protein & Present & 5.81 & $2.90-11.6$ & $<0.001$ \\
& $<150($ Ref) & 1 & & \\
Lactate dehydrogenase & $>=150$ & 1.77 & $1.09-2.85$ & 0.019 \\
Neutrophil to lymphocyte ratio & $<500$ (Ref) & 1 & & \\
& $>=500$ & 1.98 & $1.25-3.16$ & 0.004 \\
Creatinine* on admission & $>5$ (Ref) & 1 & & \\
\hline
\end{tabular}

*per unit increase

Table 3: Risk Factors associated with mortality

Variables

Died $(n=58) \quad$ Recovered $(n=360) \quad$ Univariable OR (95\% CI)

Age groups 


\begin{tabular}{|c|c|c|c|}
\hline Variables & Died $(n=58)$ & Recovered $(n=360)$ & Univariable OR $(95 \% \mathrm{CI})$ \\
\hline$<60$ years $($ Ref $)$ & 23 & 257 & 1 \\
\hline$>=60$ years & 35 & 103 & $3.79(2.14-6.74)$ \\
\hline \multicolumn{4}{|l|}{ Sex } \\
\hline Female (Ref) & 11 & 130 & 1 \\
\hline Male & 47 & 230 & $2.42(1.21-4.82)$ \\
\hline \multicolumn{4}{|c|}{ Co-morbids (present vs. not present) } \\
\hline Diabetes & 28 & 124 & $1.78(1.02-3.11)$ \\
\hline Hypertension & 31 & 124 & $2.18(1.25-3.83)$ \\
\hline Ischemic heart disease & 20 & 50 & $3.26(1.76-6.06)$ \\
\hline CKD & 12 & 17 & $5.26(2.36-11.72)$ \\
\hline Malignancy & 7 & 12 & $3.98(1.50-10.58)$ \\
\hline \multicolumn{4}{|c|}{ Radiologic findings (present vs. absent) } \\
\hline Bilateral patchy infiltrates & 52 & 224 & $5.14(2.15-12.30)$ \\
\hline \multicolumn{4}{|l|}{ Laboratory investigations } \\
\hline \multicolumn{4}{|l|}{ NLR } \\
\hline$<5$ (ref) & 15 & 227 & 1 \\
\hline$>=5$ & 43 & 133 & $4.89(2.62-9.14)$ \\
\hline \multicolumn{4}{|l|}{ Ferritin } \\
\hline$<1500$ & 18 & 217 & 1 \\
\hline$>=1500$ & 40 & 143 & $3.37(1.86-6.11)$ \\
\hline \multicolumn{4}{|l|}{ CRP } \\
\hline$<150$ (ref) & 23 & 216 & \\
\hline$>=150$ & 35 & 144 & $2.28(1.29-4.02)$ \\
\hline \multicolumn{4}{|l|}{ D-Dimer } \\
\hline$<1.5$ (Ref) & 14 & 206 & \\
\hline$>=1.5$ & 44 & 154 & $4.20(2.22-7.94)$ \\
\hline \multicolumn{4}{|l|}{ Creatinine } \\
\hline$<=1.2(\operatorname{Ref})$ & 19 & 264 & \\
\hline$>1.2$ & 39 & 96 & $5.64(3.11-10.24)$ \\
\hline \multicolumn{4}{|l|}{ Type of admission unit } \\
\hline ICU & 40 & 22 & $34.14(16.9-69)$ \\
\hline $\mathrm{SCU}$ & 17 & 123 & $0.78(0.43-1.46)$ \\
\hline Ward & 1 & 211 & $0.012(0.001-0.09)$ \\
\hline \multicolumn{4}{|l|}{ Treatment } \\
\hline NIV & 34 & 83 & $4.72(2.65-8.42)$ \\
\hline Invasive ventilation & 36 & 22 & $25.14(12.69-49.80)$ \\
\hline Oxygen support & 57 & 204 & $43.5(5.97-318.24)$ \\
\hline Systemic steroids & 50 & 187 & $5.78(2.67-12.54)$ \\
\hline Tocilizumab & 19 & 66 & $2.21(1.20-4.07)$ \\
\hline \multicolumn{4}{|c|}{ Complications present (vs absent) } \\
\hline ARDS & 51 & 62 & $35.01(15.1-80.8)$ \\
\hline Septic Shock & 46 & 10 & $134(54.89-327.9)$ \\
\hline MODS & 34 & 6 & $83.5(31.96-218.58)$ \\
\hline Nosocomial Infection & 29 & 27 & $12.33(6.46-23.55)$ \\
\hline AKI & 47 & 43 & $31.39(15.13-65.13)$ \\
\hline NSTEMI & 21 & 21 & $9.16(4.58-18.33)$ \\
\hline Mean Length of stay \pm SD & $9.64 \pm 7.83$ & $7.17 \pm 6.76$ & $1.04(1.01-1.07)$ \\
\hline
\end{tabular}

Abbreviations: CKD: Chronic kidney disease; CXR: Chest X-ray; NLR: neutrophil to lymphocyte ratio; 
CRP: C-reactive protein; ICU: Intensive care unit; SCU: Special care unit; NIV: Non-invasive ventilation; ARDS: Acute respiratory distress syndrome; MODS: Multi-organ dysfunction Syndrome; AKI: Acute kidney injury; NSTEMI: Non-ST elevation myocardial infarction

\section{LIST OF FIGURES}

Figure 1. Weekly time plot of hospital admissions according to severity of disease, showing predominance of mild cases in week 2 and 3 after the first reported case, with subsequent rise of severe cases peaking at week 11 and 12. At week 14, a week before the sharp decline over the peak of the curve, severe cases decline and there are predominantly mild cases.

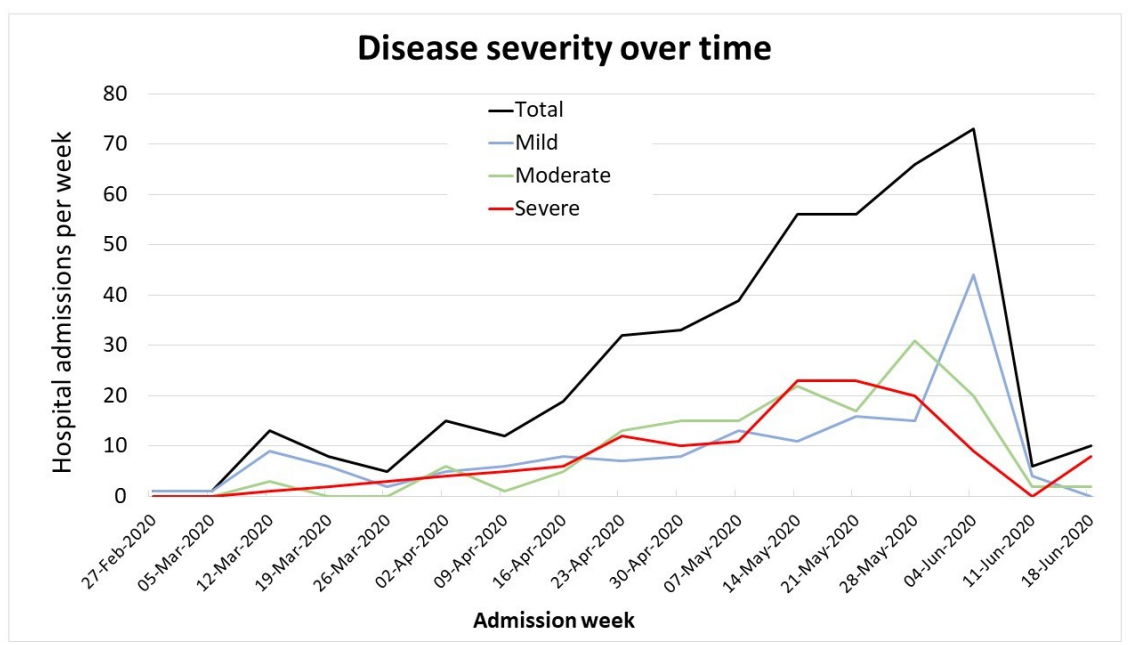

